\title{
Agua para consumo humano en Costa Rica: de los objetivos de desarrollo del milenio a los objetivos de Desarrollo Sostenible
} Drinking-water in Costa Rica: from millennium development goals to Sustainable Development goals

Darner A. Mora -Alvarado1', Carlos Felipe Portuguez-Barquero²

Mora-Alvarado, D; Portuguez-Barquero, C. Agua para consumo humano en Costa Rica: de los objetivos de desarrollo del milenio a los objetivos de Desarrollo Sostenible. Tecnología en Marcha. Diciembre 2019. Vol 32 Especial. Laboratorio Nacional de Aguas. Pág 26-36.

doi https://doi.org/10.18845/tm.v32i10.4878

1 Director del Laboratorio Nacional de Aguas. Instituto Costarricense de Acueductos y Alcantarillados. Costa Rica. Correo electrónico: dmora@aya.go.cr.

2 Gestor Ambiental. Laboratorio Nacional de Aguas. Instituto Costarricense de Acueductos y Alcantarillados. Costa Rica. Correo electrónico: fportuguez@aya.go.cr. 


\title{
Palabras clave
}

Agua; calidad; desarrollo; objetivo; servicio; sostenible.

\section{Resumen}

El presente estudio analiza los avances de Costa Rica con el paso de los "Objetivos de Desarrollo del Milenio" a los "Objetivos de Desarrollo Sostenible", usando el nuevo concepto "Agua potable gestionada en forma segura", para establecer metas al 2022 y 2030. Se utilizaron los resultados de los informes anuales de cobertura y calidad del agua del Laboratorio Nacional de Aguas (1991-2015), y el Informe de OMS/UNICEF "25 Progresos en Materia de Saneamiento y Agua Potable en el Mundo" como fuente de datos para este estudio. En cuanto a cobertura con agua suministrada por cañería, los resultados indican que Costa Rica (97\%), al utilizar el concepto de cobertura con agua de calidad potable en lugar de "Fuentes de Agua Potable Mejoradas", avanzó más que el promedio mundial (58\%) y el latinoamericano (89\%) en el periodo 19902015. Respecto a la calidad del agua, Costa Rica pasó de un 50\% de población con agua de calidad potable a un 91,2\% en el periodo de 1990-2015. Además, en 2017 la cobertura de agua potable, libre de contaminación fecal y química, fue del 93,3\%, estableciendo las metas para 2022 y 2030 del 95\% y 99\% respectivamente. Por último, se recomienda ejecutar la estrategia para mejorar la calidad de los servicios de agua potable de 1989 al 2030, con el fin de alcanzar la universalización del suministro de los servicios de agua potable en Costa Rica.

\section{Keywords}

Water; quality; development; objective; service; sustainable.

\begin{abstract}
The present study analyzes the progress made in Costa Rica regarding "Clean water and Sanitation", since the implementation of the Millennium Development Goals from 1990 to 2015 and the Sustainable Development Goals from 2016 to 2030. The results from the annual reports of drinking-water coverage and quality made by the Laboratorio Nacional de Aguas (19912015), and data from the WHO/UNICEF report "25 Progress on Drinking-water, Sanitation and Hygiene" were used as source of information data for this study. In therms of coverage with water supplied by pipe, the results indicate that Costa Rica (97\%) has made more progress than the rest of the world (58\%) and Latin America (89\%) within the period 1990-2015. Regarding water quality, Costa Rica went from $50 \%$ of the population with water of potable quality to $91.2 \%$ within the period of 1990-2015. Additionally, in 2017 the coverage of potable drinking-water -free from faecal and chemical pollution- was $93.3 \%$, stablishing the goals for 2022 and 2030 of $95 \%$ and $99 \%$ respectively. Lastly, this study recommends to carry out the strategy to improve the quality of drinking-water services from 1989 to 2030, in order to universalize the water supply in Costa Rica.
\end{abstract}

\section{Introducción}

En setiembre del año 2000 representantes de 189 países se dieron cita en la "Cumbre del Milenio", realizada en Nueva York, para adoptar los Objetivos de Desarrollo del Milenio (ODM), promovidos por la Organización Mundial de la Salud (OMS) y el Fondo de las Naciones Unidas para la Infancia (UNICEF); los mismos tenían como propósito emprender una acción consensuada para mejorar el ambiente y la salud pública(1). Para medir el avance en las coberturas de acceso con agua potable en cada país, los mencionados organismos 
internacionales crearon el "Programa Conjunto de Monitoreo" (PCM) ${ }^{(2)}$. La meta del ODM 7 , impulsa a los países a "Reducir para el 2015, la proporción de personas, sin acceso sostenible a agua potable y saneamiento".

Para efectos del presente estudio se aborda el acceso o cobertura con "Fuente mejorada de agua potable", la cual fue definida como "una que por la naturaleza de su construcción a través de una intervención activa está protegida de la contaminación externa, en particular de la contaminación con materia fecal". Para permitir la comparación internacional de los datos, se han clasificado distintos tipos de fuentes de agua tanto en "Fuentes mejoradas" como en "Fuentes no mejoradas" (3). Dicho sistema de clasificación toma en cuenta características geológicas propias de la fuente, características estructurales de la toma de agua y aspectos relacionados con el sistema de distribución.

Utilizando esta clasificación, 189 países de la "Cumbre del Milenio" realizaron una estimación de la línea base de calidad del agua para 1990 a 2015. El análisis Ilevado a cabo indicó que, a nivel global, los países pasaron de un 76\% de cobertura con agua potable en 1990 a un

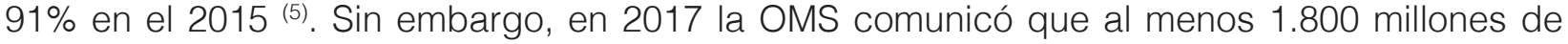
personas, con acceso a fuentes de agua potable mejoradas, utilizaban agua que presentaba contaminación fecal, lo que significaba un riesgo para la salud de los usuarios ${ }^{(6)}$.

En 2015, la OMS formuló los nuevos "Objetivos de Desarrollo Sostenible" (ODS) ${ }^{(7)}$. En el número seis "Agua Limpia y Saneamiento" (8), se plantea el objetivo de "Garantizar la disponibilidad a agua, su gestión sostenible y el saneamiento para todos, específicamente lograr para al 2030 el acceso universal y equitativo al agua potable, a un precio asequible para todos". En razón de la comprobación de las deficiencias detectadas en el concepto "Fuentes mejoradas de agua potable", la OMS y el PCM lo sustituyeron por un nuevo término denominado "Agua potable gestionada en forma segura" (9), que se define como "Agua para consumo proveniente de una fuente mejorada, ubicada dentro de la vivienda o en el patio o parcela, disponible en todo momento, necesario y libre de contaminación fecal y sustancias químicas" tóxicas prioritarias (flúor y arsénico).

A nivel nacional, el Laboratorio Nacional de Aguas (LNA) prepara informes anuales de cobertura y calidad del agua, en donde se realizan estimaciones sobre el suministro de agua por cañería intradomiciliar y de su calidad ${ }^{(10,11)}$, fundamentados en resultados de análisis microbiológicos y físico-químicos, que responden a las exigencias de las diferentes versiones del "Reglamento para la Calidad del Agua Potable". $(12,13,14,15)$. Por otro lado, también estableció los datos líneabase para los años 2016 y 2017, sobre la cobertura y calidad del agua para consumo humano $(\mathrm{ACH})$ en viviendas y más allá del hogar, en centros educativos y en centros de salud $(16,17,18)$. Fundamentados en la evolución de los conceptos de la OMS, este estudio tiene como objetivo presentar la variación de los datos de cobertura y la calidad del ACH en Costa Rica, con la sustitución del Objetivo 7 de los ODM por el Objetivo 6 de los ODS, entre el periodo 1990-2015, los datos línea-base 2016-2017, y las metas establecidas para los años 2022 y 2030.

\section{Metodología}

Coberturas del suministro de agua como fuentes de agua potable en Costa Rica: 1990-2015.

\section{Datos de OMS/UNICEF}

Con base en este antiguo concepto, mediante el informe "25 Progresos en Agua Potable y Saneamiento del 2015" de OMS/UNICEF, se estimó el avance, en términos de promedio de cobertura del $\mathrm{ACH}$ con "Fuentes de agua potable mejoradas" del mundo, América Latina y El Caribe y Costa Rica, del periodo 1990-2015. 
Cobertura y calidad del agua en Costa Rica según LNA 1990-2015

La cobertura y calidad del ACH se obtuvo de 2 informes anuales realizados por el LNA en 1991 y 2015: Situación Actual del Agua para Consumo Humano y Aguas Residuales en Costa Rica; 1991 (19); y Agua para Consumo Humano y Saneamiento en Costa Rica al 2016: metas al 2022 y 2030 (20).

Calidad del agua en Costa Rica al año 2017

Con el informe titulado "Agua Potable y Saneamiento: coberturas en viviendas y más allá del Hogar en Costa Rica al 2017", se definieron los datos línea-base y la escalera de los servicios de abastecimiento de agua potable en Costa Rica al 2017.

Objetivo de Desarrollo Sostenible: "Escalera del Agua" potable gestionada en forma segura

El ODS 6 "Agua Limpia y Saneamiento", además de establecer el nuevo concepto "Agua potable gestionada en forma segura" estableció la "Escalera del Agua Potable Doméstica". Esta escalera clasifica el nivel de servicio en: gestionado de forma segura, básico, limitado, no mejorado y sin servicio ${ }^{(19)}$

Metas de cobertura y calidad del agua para consumo humano al 2022 y 2030 en Costa Rica Las metas de cobertura y calidad del agua potable se establecieron con base en los datos línea-base del informe indicado en el punto 2.3. Dichas metas se dividen en dos periodos: 2017-2022 y 2023-2030.

Evolución y expectativas del suministro de agua de los

ODM (1990-2015) a los ODS (2017-2030)

Mediante la aplicación de los datos y la escalera de cobertura (ODM) y de calidad de los ODS 6 , se presenta mediante figuras y gráficos la evolución desde 1990 al 2015, datos línea-base 2017 y las expectativas con las metas propuestas para el 2022 y 2030.

\section{Resultados}

Cobertura con Fuentes de Agua Potable Mejoradas: 1990-2015

\section{Según OMS/UNICEF}

En el cuadro 1 e presentan las coberturas mediante fuentes de agua potable mejoradas en el mundo, América Latina y Costa Rica, durante los años 1990 y 2015.

Estimación de escaleras de "Fuentes de Agua Potable Mejoradas" 1990-2015

De conformidad con lo indicado por OMS/UNICEF a través del PCM, respecto a la clasificación de los servicios según los ODM, en la figura 1 se presenta la comparación de las escaleras de agua para consumo humano para los años 1990 y 2015, correspondientes al mundo, América Latina y Costa Rica.

\section{Según el LNA}

Mientras que el PCM solamente medía el avance de cobertura por cañería, pozos y nacientes, mediante el concepto de "Fuente de Agua Potable Mejoradas", el LNA estimaba la cobertura de población que recibía agua de calidad potable, a través del "Programa de Vigilancia y la Calidad del Agua para Consumo Humano". Para el año 1990, el LNA estimó que el 97,4\% de la población total del país fue abastecida a través de alguno de los entes operadores oficiales 
(AyA, municipios, ESPH, Acueductos Rurales), más agua con fácil acceso a pozos, nacientes y otros; por su parte, 93,4\% recibió agua por cañería y el 50\% fue abastecida con agua de calidad potable. El informe anual del LNA para el año 2015, permitió estimar que el 98\% de la población total recibió agua por cañería intradomiciliar, mientras que el porcentaje de población abastecida con agua de calidad potable ascendió a 91,2\%.

Cuadro 1. Porcentaje de cobertura del mundo, América Latina y Costa Rica con Fuentes de Agua Potable Mejoradas 1990-2015

\begin{tabular}{|c|c|c|c|c|c|c|}
\hline Zona & Año & Total Mejoradas & $\begin{array}{c}\text { Agua por } \\
\text { cañería }\end{array}$ & $\begin{array}{c}\text { Otras fuentes } \\
\text { Mejoradas }\end{array}$ & $\begin{array}{c}\text { Fuentes No } \\
\text { protegidas }\end{array}$ & $\begin{array}{c}\text { Agua } \\
\text { superficial }\end{array}$ \\
\hline \multirow{2}{*}{ Mundo } & 1990 & 76 & 44 & 32 & 17 & 7 \\
\cline { 2 - 7 } & 2015 & 91 & 58 & 33 & 7 & 2 \\
\hline \multirow{2}{*}{$\begin{array}{c}\text { América } \\
\text { Latina }\end{array}$} & 1990 & 85 & 73 & 12 & 8 & 7 \\
\cline { 2 - 7 } & 2015 & 95 & 89 & 6 & 4 & 1 \\
\hline \multirow{2}{*}{ Costa Rica } & 1990 & 93 & 83 & 10 & 3 & 4 \\
\cline { 2 - 7 } & 2015 & 98 & 97 & 1 & 1 & 1 \\
\hline
\end{tabular}

Fuente: OMS/UNICEF: 2015

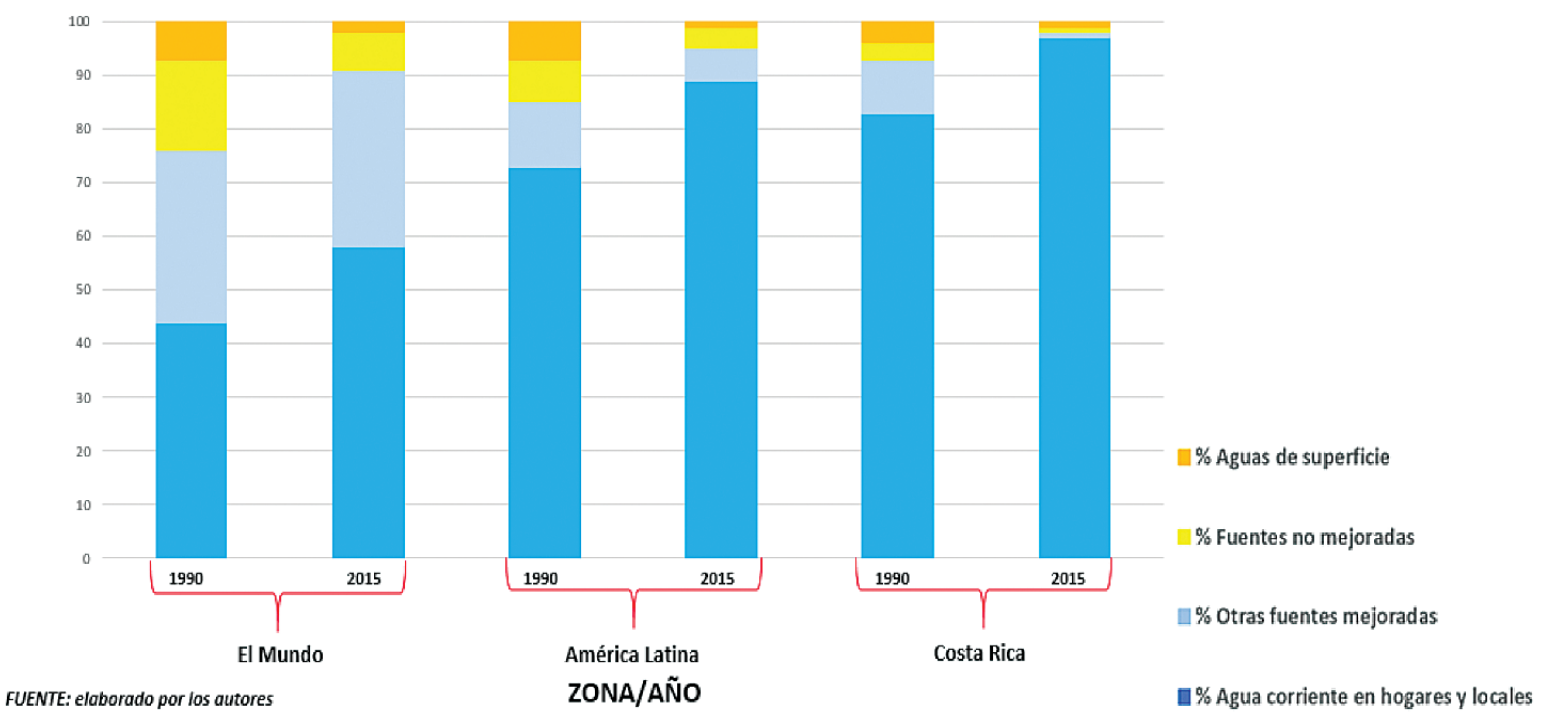

Figura 1. Escalera de agua de Cobertura con FAPM según ODM en el mundo, América Latina y Costa Rica: 19902015.

Cobertura y calidad del agua para consumo humano 2017

En el cuadro 2 se presentan los datos de cobertura y calidad del agua para el año 2017. Los mismos permitieron calcular el porcentaje de población abastecida con "Agua potable gestionada en forma segura", además de los otros tipos de servicios indicados en la "Escalera de Agua" potable doméstica de los ODS. 
Cuadro 2. Agua para consumo humano: estimación general de cobertura y calidad en Costa Rica - periodo 2017.

\begin{tabular}{|c|c|c|c|c|c|c|c|c|c|}
\hline \multirow[t]{2}{*}{ Abastecimiento } & \multirow{2}{*}{$\begin{array}{c}\mathrm{N}^{\circ} \\
\text { Acueductos }\end{array}$} & \multicolumn{2}{|c|}{$\begin{array}{l}\text { Población } \\
\text { cubierta }\end{array}$} & \multicolumn{2}{|c|}{$\begin{array}{l}\text { Población con } \\
\text { agua potable }\end{array}$} & \multicolumn{2}{|c|}{$\begin{array}{c}\text { Población con } \\
\text { agua No Potable }\end{array}$} & \multicolumn{2}{|c|}{ Acueductos } \\
\hline & & Población & $\%$ & Población & $\%$ & Población & $\%$ & Pot. & No Pot. \\
\hline AyA & 204 & 2.325 .452 & 47,0 & 1.302 .198 & 99,0 & 23.254 & 1,0 & 173 & 31 \\
\hline Municipalidades & 237 & 623.057 & 12,6 & 593.151 & 95,2 & 29.906 & 4,8 & 217 & 20 \\
\hline $\mathrm{ESPH}$ & 14 & 224.665 & 4,6 & 224.665 & 100 & 0 & 0 & 14 & 0 \\
\hline CAAR'IASADAS * & 956 & 996.143 & 20,1 & 847.718 & 85,1 & 148.425 & 14,9 & 718 & 238 \\
\hline CAAR'/ASADAS ** & 1.267 & 583.644 & 11,8 & 496.681 & 85,1 & 86.963 & 14,9 & 952 & 315 \\
\hline $\begin{array}{c}\text { Subtotal por entidad } \\
\text { operadora }\end{array}$ & 2.678 & 4.752 .961 & 96,1 & 4.464 .413 & 94,0 & 288.548 & 6,0 & 2.074 & 604 \\
\hline 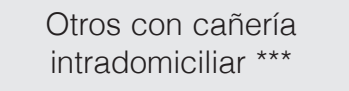 & ND & 22.081 & 0,4 & 20.756 & 94,0 & 1.325 & 6,0 & ND & ND \\
\hline $\begin{array}{l}\text { Otros con agua por } \\
\text { cañería en el patio *** }\end{array}$ & ND & 151.865 (1) & 3,1 & 1421.753 & 94,0 & 9.112 & 6,0 & ND & ND \\
\hline $\begin{array}{l}\text { Subtotal de población } \\
\text { abastecida por cañería } \\
\end{array}$ & 2.678 & 4.926 .907 & 99,6 & 4.627 .922 & 94,0 & 298.985 & 6,0 & 2.074 & 604 \\
\hline $\begin{array}{l}\text { Sin tubería: pozos- } \\
\text { nacientes }\end{array}$ & ND & $19.793(1)$ & 0,4 & 18.605 & 94,0 & 1.188 & 6,0 & ND & ND \\
\hline TOTALES & 2.678 & $\begin{array}{l}4.946 .700 \\
(1)\end{array}$ & 100 & 4.646 .527 & 93,9 & 300.173 & 6.1 & 2.074 & 604 \\
\hline
\end{tabular}

Fuente: LNA e INEC.

ND: no determinado.

(1) Población estimada por el INEC con la ENAHO julio 2017.

* Evaluados en el periodo 2015 al 2017, con un 85,1\% de población con agua potable.

** De acuerdo a la metodología, se aplica el $85,1 \%$ obtenido en los acueductos evaluados.

${ }^{* * \star}$ Se aplica el $94,0 \%$ obtenido en el subt

En el primer trimestre del año 2018, el LNA estimó las coberturas de los servicios de agua potable gestionados en forma segura del año 2017 para viviendas, centros educativos y centros de salud. Estos datos sirvieron para establecer una línea-base de calidad, que permitieron proponer las metas para el 2022 y 2030 del ODS 6 "Agua Limpia y Saneamiento, en el marco de los "Objetivos de Desarrollo Sostenible". En este sentido, los datos línea-base se presentan a continuación en el cuadro 3 mediante la aplicación de la "Escalera del Agua":

\section{Metas en el suministro de agua potable al 2022 y 2030 en Costa Rica}

El cuadro 4 resume la situación actual de línea-base de calidad de los servicios de agua potable para los años 2016-2017, y las metas planteadas para los años 2022 y 2030. 
Cuadro 3. Aplicación de la "Escalera del Agua Potable" en Costa Rica (periodo 2017)

\begin{tabular}{|c|c|c|c|}
\hline $\begin{array}{c}\text { Agua potable gestionada } \\
\text { de forma Segura }\end{array}$ & $\begin{array}{r}\text { Fuente mejorada ubicada en las instalaciones, "disponible cuando } \\
\text { sea necesario", y libre de contaminación. }\end{array}$ & $\begin{array}{r}94,0 \%{ }^{*} \\
\text { Servicio básico }\end{array}$ & $\begin{array}{r}\text { Fuentes de agua mejoradas en } 30 \text { minutos, incluido ida y vuelta, o en } \\
\text { las instalaciones pero con contaminac ón. }\end{array}$ \\
\hline Servicio limitado & Fuente de agua mejorada que consigue agua por encima de 30 \\
minutos, incluido ida y vuelta. & $0,0 \%$ \\
\hline Servicio no mejorado & Fuente de agua no mejorada que no protege de la contaminación. & $0,4 \%$ \\
\hline Sin servicio & Agua superficial. & $0,0 \%$ \\
\hline
\end{tabular}

Nota : *dato de 93,9\% redondeado a $94,0 \%$.

Fuente : elaborado por los autores.

Cuadro 4. Actividades y Metas de Calidad de los Servicios de Agua Potable para los Periodos $2016-2017$ y Metas para el 2022 y 2030

\begin{tabular}{|c|c|c|c|c|}
\hline Actividad & Indicador & $\begin{array}{l}\text { Situación actual } \\
\text { 2016-2017 }\end{array}$ & Meta 2022 & Meta 2030 \\
\hline Cobertura de agua por cañería & $\begin{array}{l}\text { Porcentaje } \\
\text { cobertura }\end{array}$ & $99.5-99.6$ & 99.9 & 100 \\
\hline $\begin{array}{l}\text { Cobertura de agua por cañería } \\
\text { intradomiciliar }\end{array}$ & $\begin{array}{l}\text { Porcentaje } \\
\text { cobertura }\end{array}$ & $99.5-99.6$ & 98.5 & 100 \\
\hline $\begin{array}{c}\text { Cobertura de población con agua } \\
\text { de calidad potable }\end{array}$ & $\begin{array}{l}\text { Porcentaje } \\
\text { cobertura }\end{array}$ & $91.8-93.9$ & 95 & 99 \\
\hline $\begin{array}{l}\text { Cobertura de población con agua } \\
\text { sometida a control de calidad }\end{array}$ & $\begin{array}{l}\text { Porcentaje } \\
\text { población }\end{array}$ & $74.1-73.6$ & 85 & 99 \\
\hline $\begin{array}{l}\text { Cobertura de población con agua } \\
\text { con tratamiento/desinfección }\end{array}$ & $\begin{array}{l}\text { Porcentaje } \\
\text { población }\end{array}$ & $86.3-88.8$ & 95 & 99 \\
\hline $\begin{array}{l}\text { Acueductos con Planes de } \\
\text { Seguridad del Agua }\end{array}$ & $N^{\circ}$ acueductos & $75^{\star \star}$ & 850 & 1.250 \\
\hline $\begin{array}{l}\text { Acueductos con agua de calidad } \\
\text { potable }\end{array}$ & $N^{\circ}$ acueductos & $1.878-2.074$ & $2.200^{*}$ & $2.562^{\star}$ \\
\hline $\begin{array}{l}\text { Porcentaje de acueductos con } \\
\text { agua de calidad potable }\end{array}$ & $\mathrm{N}^{\circ}$ acueductos & $72.9-77.4$ & 85 & 99 \\
\hline $\begin{array}{l}\text { Acueductos con Programa Sello } \\
\text { Calidad de Sanitaria (PSCS) }\end{array}$ & $N^{\circ}$ galardonados & $563^{\star \star \star}$ & $845(50 \%)$ & $1.226(100 \%)$ \\
\hline $\begin{array}{c}\text { Población con agua sometida a } \\
\text { PSCS }\end{array}$ & $\begin{array}{c}\text { Población } \\
\text { con agua de } \\
\text { acueductos con } \\
\text { PSCS }\end{array}$ & $\begin{array}{c}2.430 .709^{\star *} \\
(49,7 \%)\end{array}$ & $\begin{array}{c}3.649 .361 \\
(70.0 \%)\end{array}$ & $\begin{array}{l}4.420 .013 \\
(80 \%)\end{array}$ \\
\hline
\end{tabular}

*Se toma comobase los datos del 2016 y 2017 de 2.547 y 2.678 acueductos monitoreados, respectivamente. Este valor aumenta conforme se incremente el inventario anual de acueductos.

${ }^{\star \star}$ Dato del año 2016. 
Evolución y espectativas del suministro de agua de los ODM (1990-2015) a los ODS 2017-2022 y 2023-2030

En la figura 2 se presenta evolución y las expectativas de los servicios de agua potable de los ODM (1990-2015) a los ODS (2022 y 2030), y según la clasificación de la "Escalera del Agua" propuesta en los ODS.

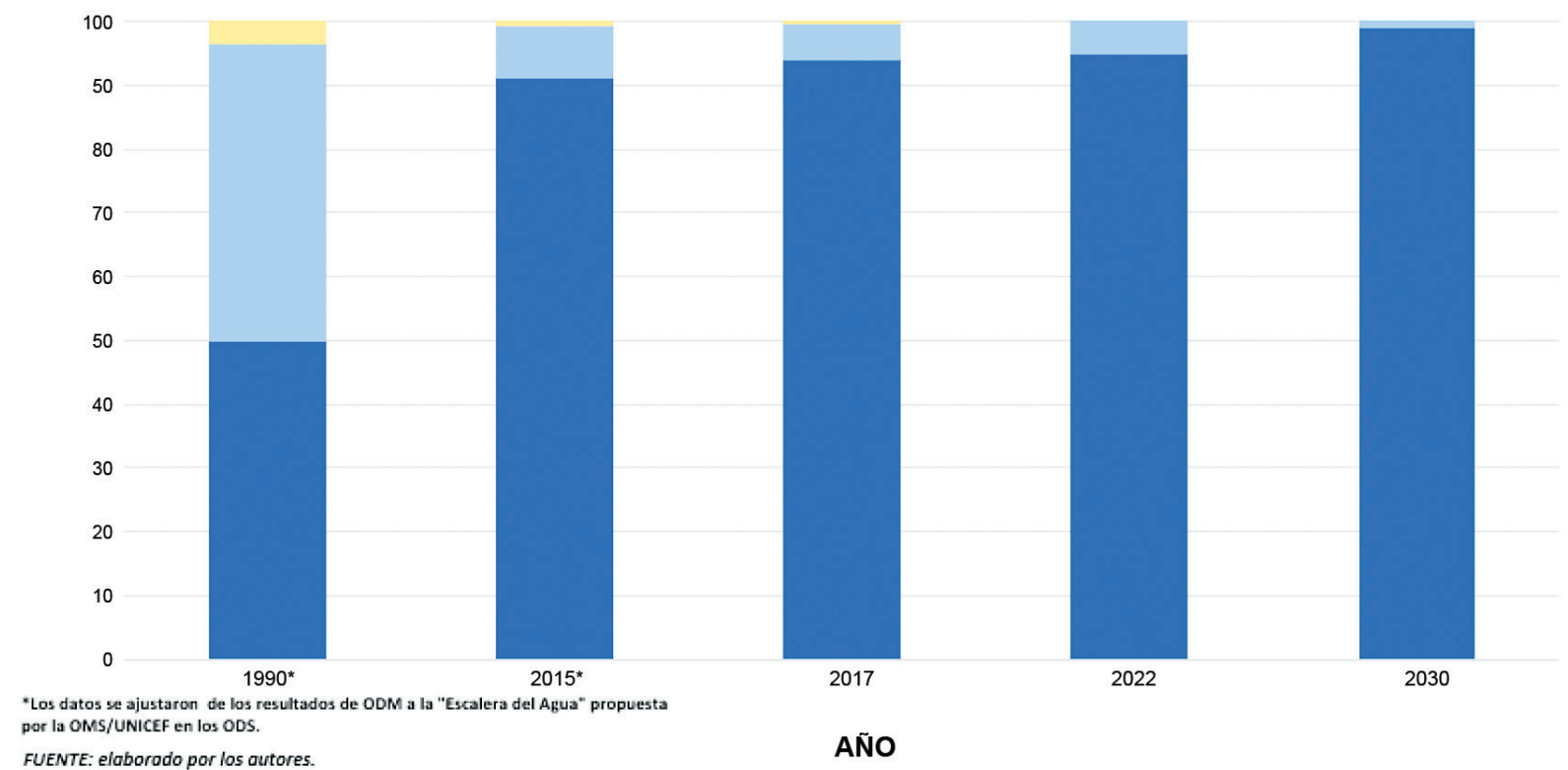

Figura 2. Escalera del Agua para Consumo Humano según ODS en Costa Rica. Periodos 1990, 2015, 2017, 2022 y 2030.

\section{Análisis de resultados}

La decisión del LNA de realizar informes anuales de cobertura y calidad del ACH suministrada en Costa Rica, ha permitido no solo valorar los avances en cobertura bajo el concepto de la OMS/UNICEF de "Fuentes de Agua Potable Mejorada", sino también las coberturas de población que recibió agua de calidad potable por cañería intradomiciliar, determinada así a través de análisis físico-químicos y microbiológicos. Es decir, Costa Rica ha sido uno de los pocos países en el mundo que reportó el porcentaje de "Fuentes de Agua Potable Mejorada" como sinónimo de agua de calidad potable, para el periodo 1990-2015.

\section{Avance de Costa Rica en "Fuentes de Agua Potable Mejoradas"}

Estimacion de la escalera de Fuentes de Agua Potable Mejoradas: 1990-2015

Como se observa en el cuadro 1 y figura 1, Costa Rica logró avanzar en el suministro de agua por cañería de un 83\% en 1990 a 97\% en el 2015, mientras que el mundo pasó de un 44\% a 58\% y América Latina del 73\% a 89\%, en el mismo periodo. Estos datos, reflejan las aplicaciones de las escaleras de acceso a Fuentes Mejoradas de Agua Potable en el mundo, América Latina y Costa Rica, lo cual permite observar un avance superior en nuestro país de 39\% con respecto al mundo en el suministro de agua por cañería y $8 \%$ con respecto a América Latina. 
Cobertura y calidad del agua para consumo humano 1990 y 2015 según el LNA

Según el "Programa de Vigilancia y Control de Calidad del Agua para Consumo Humano" del LNA, se estimó que: El país pasó de 93,4\% a 97,4\% de agua suministrada por cañería entre los años 1990 y 2015; y la cobertura de población cubierta con agua de calidad potable para 1990 se estimó en 50\%, y para el 2015 alcanzó el 91,2\%.

Datos línea-base en agua potable gestionada en forma segura

Con los datos del informe anual del LNA al 2017, se estimó que Costa Rica logró alcanzar de agua por cañeria intradomiciliar de 96,1\% y 3,5\% por cañería fuera de la vivienda, para un total de 99,6\% por todo tipo de cañería. Además, alcanzó la cobertura máxima de agua de calidad potable del 93,9\%.

Metas de servicios de agua potable gestionados en forma segura al 2022 y al 2030

Las metas de agua sumistrada por "Servicios de Agua Potable Gestionado en Forma Segura", indican que en el año 2022 Costa Rica debe alcanzar un 95\% y en 2030 entre 99\% y el 100\% de cobertura. Para lograr estas metas, es necesario la aplicación de lo estipulado en la "Política Nacional en Agua Potable" (21), incorporar los centros educativos y de salud en la vigilancia y control de calidad del agua, y establecer o ampliar el "Programa Nacional de Mejoramiento y Sostenibilidad de la Calidad de los Servicios de Agua Potable 2017-2030" (22). Por otro lado, para cumplir con parte del ODS 6, es fundamental estimar el dato del servicio de agua potable, complementado con el de calidad y continuidad de los servicios, establecidos en el documento titulado "Índice de Calidad y Continuidad de los Servicios de Agua Potable en Costa Rica" (23).

Evolución y expectativas del suministro de agua potable de los ODM (1990-2015) a los ODS (2017-2030)

La evolución de la cobertura y calidad del $\mathrm{ACH}$ en Costa Rica ha estado precedida por diferentes situaciones como: la creación del "Servicio Nacional de Acueductos y Alcantarillados" (SNAA) en 1961 (24); la creación del "Programa Nacional de Acueductos Rurales" en 1966; y la Implementación de la estrategia para mejorar la calidad de los servicios de agua potable entre 1989 y el 2030, la cual incluye los siguientes aspectos:

- Elaboración de informes de cobertura y calidad del agua para consumo y saneamiento por parte del LNA.

- En 1991 se creó el "Código de Colores", para medir el avance de la calidad del agua por acueducto.

- Conformación de comités locales dentro del marco del "Programa Bandera Azul Ecológica", creado en el Laboratorio Central del AyA en el año 1996.

- Se promovió y ejecutó la designación del Laboratorio Central del AyA como Laboratorio Nacional de Aguas (LNA), Decreto Ejecutivo 26066-S del mes de junio del 1997.

- En 2002 se implementó la iniciativa del "Programa Sello de Calidad Sanitaria" (PSCS).

- Del 2002 al 2006 se implementó el "Programa Nacional de Mejoramiento de la Calidad del Agua Potable" (PNMCAP), con los componentes: "Protección de Fuentes", "Vigilancia y Control de Calidad del Agua", "Tratamiento y/o Desinfección", "Normalización y Legislación", "Evaluación de Riesgo en los Acueductos" y "Autosostenibilidad, Movilización Social y Educación".

- En el 2007 se estableció, vía Decreto 33953-S-MINAE, el "Programa Nacional de Mejoramiento y Sostenibilidad de la Calidad de los Servicios de Agua Potable 2007-2015" (PNMSCSAP) ${ }^{(25)}$. 


\section{Conclusiones}

La cobertura con ACH de 1990 y 2015, indica que Costa Rica pasó de 83\% a 97\%, lo que superó el promedio mundial de 44\% a 58\%, y de América Latina de un 73\% a 89\%.

Costa Rica prácticamente no aplicó el concepto de "Fuentes de Agua Potable Mejoradas" de la OMS/UNICEF, y en su lugar utilizó la cobertura de agua de calidad potable, avanzando de un $50 \%$ en 1990 a un $91,2 \%$ en el 2015.

A los datos de calidad del agua de Costa Rica de 1990 y 2015 se les aplicó la herramienta de la "Escalera del Agua Potable Doméstica", para evolucionar de los ODM a los ODS, y así poder compararlos con los resultados del 2017 y las metas planteadas al 2022 y 2030, como lo muestra la figura 2.

El paso del dato de suministro de ACH de los ODM (1990-2015) a los ODS $(2015$, ) indican que Costa Rica podría alcanzar la universalización del suministro de agua gestionada en forma segura en el 2030, siempre y cuando se cumpla con los lineamientos de la Política Nacional de Agua Potable, y la estrategia establecida por el LNA desde 1989 y hasta el 2017, y su ampliación al 2030.

\section{Recomendaciones}

Complementar el dato de la calidad del agua con el parámetro de continuidad del servicio, para lo cual es fundamental aplicar el "Índice de Calidad y Continuidad del Servicio", elaborado por el LNA y el Área Operativa de AyA en el año 2017.

Continuar con la elaboración de los Informes de Cobertura y Calidad del Agua para Consumo y Saneamiento en Centros Educativos y Centros de Salud en Costa Rica, además de ampliar la estrategia para mejorar la calidad de los servicios de agua potable al año 2030, y más allá.

\section{Referencias}

[1] Barboza K; Ortega W. 2015. Objetivos de Desarrollo del Milenio. III Informe país 2015. San José, Costa Rica. MIDEPLAN; PAG 1-99.

[2] Organización Panamericana de la Salud. 2011. Agua y Saneamiento. Washington, D.C.20037, EUA; OPS; pag 1-65.

[3] OMS/UNICEF. Preguntas fundamentales sobre agua potable y saneamiento para encuestas de Hogar. OMS/ UNICEF; JMP. www. wssinfo.org.

[4] OMS. 2004. Guias de Calidad del Agua Potable. Tercera Edición. Volumen 1. Recomendaciones. OMS; Ginebra, Suiza; Pag 90-92.

[5] OMS/UNICEF. 2015. 2017. 25 Progresos en materia de saneamiento y agua potable. Informe de actualización 2015 y evaluación de ODM. Ginebra 27, Suiza. Impr.en Nueva York; sp.

[6] OMS. 2017. 1800 millones de personas en todo el mundo utilizan fuentes de agua con contaminación fecal. OMS; En: amp.infosalus.com

[7] OMS. Objetivos de Desarrollo Sostenible. Documento en linea. ONU; www. undp.org $>$ content $>$ undp $>$ home.

[8] PNUD. Costa Rica. Objetivo 6. Agua Limpia y Saneamiento. PNUD; En línea. www.cr.undp.org.

[9] OMS/UNICEF. 2017. Agua potable gestionada en forma segura. OMS/UNICEF; Nueva York; pag 53.

[10] Mora D; Portuguez C. 2002. Situación de cobertura y calidad del agua para Consumo Humano y dispocisión de excretas en Costa Rica a finales del año 2001. La Unión, Cartago. Laboratorio Nacional de Aguas; sp.

[11] Mora D; Mata A; Portuguez C. 2016. Agua para Consumo Humano y Saneamiento y su relación con los indicadores básicos de Salud en Costa Rica: Objetivos de Desarrollo del Milenio y la Agenda para el 2030. La Unión, Cartago. Laboratoiro Nacional de Aguas; Pag 1-19.

[12] Comité Coordinador Regional de Instituciones de Agua Potable y Saneamiento de Centroamérica, Panamá y República Dominicana. 1995. Normas de Calidad del Agua para Consumo Humano. San José, Normas CAPRE; pag: 1-17. 
[13] Poder Ejecutivo. 1997. Reglamento para la Calidad del Agua Potable. San José, Costa Rica. La Gaceta №100 del 27 de mayo. Decreto Ejecutivo No25991-S; pag: 1-3.

[14] Poder Ejecutivo. 2005. Reglamento para la Calidad del Agua Potable. San José, Costa Rica. Decreto Ejecutivo 32327-S; La Gaceta №84 del 03 de mayo; Pag 1-16.

[15] Poder Ejecutivo. 2015. Reglamento para la Calidad del Agua Potable. San José. Costa Rica. Decreto Ejecutivo 38924-S. La Gaceta №170 del 01 de setiembre.

[16] Darner A. Mora; Carlos F. Portuguez B. Agua Potable y Saneamiento: Cobertura en Vivienda y más allá del Hogar en Costa Rica al 2017. La Unión, Cartago.Laboratorio Nacional de Aguas; 2017: pag 1-28.

[17] Darner A.Mora; Pablo Rivera; Flora Acuña; Carlos F. Portuguez. Agua para Consumo Humano y Saneamiento en Centros Educativos de Costa Rica al año 2017. La Unión, Cartago. Laboratorio Nacional de Aguas; 2018: pag 1-12.

[18] Darner A. Mora; Pablo Rivera. Estimación de Calidad del Agua para Consumo Humano en Centros de Salud de Costa Rica al año 2017. La Unión, Cartago, Laboratorio Nacional de Aguas; 2018: pag 1-17.

[19] Darner A. Mora. Situación actual del agua de consumo humano y las aguas residuales en Costa Rica 1994. San José, Revista Biocensos; Vol 7. N², junio 1991: pag 74-81.

[20] Darner A. Mora; Carlos F. Portuguez. Agua para Consumo Humano y Saneamiento en Costa Rica al 2016: metas al 2022 y 2030. La Unión, Cartago. Laboratoiro Nacional de Aguas; 2017: Pag 1-19.

[21] Instituto Costarricense de Acueductos y Alcantarillados; Ministerio de Salud Pública; Ministerio de Ambiente y Energía. Política Nacional para el Subsector de Agua Potable de Costa Rica; 2017-2030. AyA/MS/MINAE; San José, Costa Rica, AyA; 2017.

[22] Darner A. Mora; Carlos F. Portuguez. Programa Nacional de Mejoramiento y Sostenibilidad de la Calidad de los Servicios de Agua Potable: 2017-2030. La Unión, Cartago. Laboratoiro Nacional de Aguas; 2017: Pag 1-105.

[23] Laboratorio Nacional de Aguas. Indice de Calidad y Continuidad de los Servicios de Agua Potable en Costa Rica. La Unión, Cartago. Laboratorio Nacional de Aguas; 2018: pag 79.

[24] Poder Ejecutivo. Creación del Servicio Nacional de Acueductos y Alcantarillados. San José, Costa Rica. Decreto Ejecutivo No2727 del 14 de abril de 1961.

[25] Darner A. Mora; Héctor Feoli. Programa Nacional de Mejoramiento y Sostenibilidad de la Calidad de los Servicios de Agua Potable: 2007-2015. La Unión, Cartago. Laboratorio Nacional de Aguas; 2007: Pag 1-56. 